\title{
SARCOIDOSIS WITH INVOLVEMENT OF LARGE VESSELS
}

Vinicius Verlangieri Soubihe ${ }^{1, \star}$, Diego de Paula Ferreira Nunes ${ }^{1}$, Raíssa Dudienas Domingues Pereira ${ }^{1}$, Caio Rustichelli Cardoso ${ }^{1}$, Zoraida Sachetto ${ }^{1}$

1.Universidade Estadual de Campinas, Campinas (SP), Brazil.

*Corresponding author: vinisoubihe@uol.com.br

\section{BACKGROUND}

Sarcoidosis is a systemic granulomatous disease that usually involves lungs, thoracic and peripheral lymph nodes; however, skin, eyes, bones, liver, spleen, heart, vessels, upper respiratory tract and nervous system can also be affected. Diagnosis is confirmed when clinical and radiological findings are complemented by histological evidence of non-necrotizing granuloma.

\section{CASE REPORT}

Female, 32 years old, being followed up with a diagnosis of sarcoidosis since 2005. Initially, she had hilar adenopathy, weight loss, asthenia and lesion in the right fibula with bone biopsy showing non-necrotizing granuloma. During evolution, she presented involvement of large vessels (ascending aortic aneurysm, stenosis of right pulmonary artery, right and left subclavian artery, right common carotid artery and superior mesenteric artery). She developed pulmonary aspergilloma as an infectious complication and was submitted to right pneumectomy. Due to large vessel vasculitis, she used oral cyclophosphamide (suspended due to hemorrhagic cystitis), azathioprine and methotrexate with progression of vascular involvement and in October 2019 started infliximab associated with corticosteroid therapy. In August 2020, the patient started with diffuse abdominal pain of strong intensity and computed tomography (CT) angiography of the aorta showing an increase in her thickness and a significant stenosis of the inferior mesenteric artery that did not exist in previous exams (Figs. 1 and 2) with an increase in erythrocyte sedimentation rate and C-reactive protein.

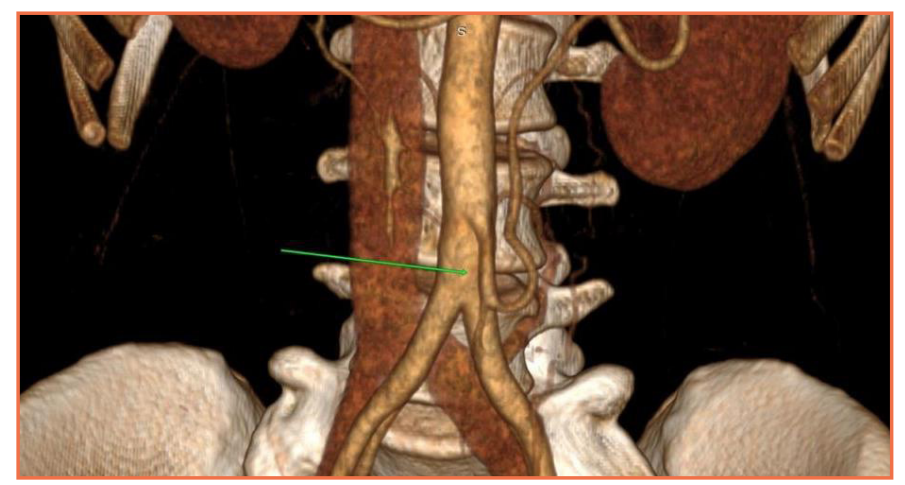

Figure 1. Arrow showing mesenteric inferior artery in CT angiography from 2019.

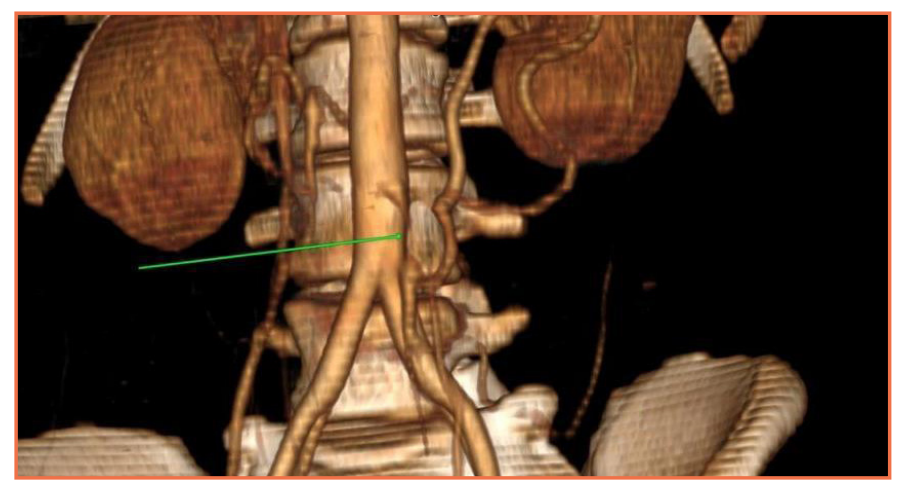

Figure 2. Arrow showing significant stenosis of mesenteric inferior artery in CT angiography from august 2020 . 
Intravenous methylprednisolone $(3 \mathrm{~g})$ was followed by prednisone $1 \mathrm{mg} / \mathrm{kg} /$ day. Then, angioplasty was performed on the affected artery (Figs. 3 and 4) with stent placement. Patient evolved with progressive improvement of symptoms and was discharged seven days after the procedure, using double antiplatelet therapy, prednisone and anticoagulation.

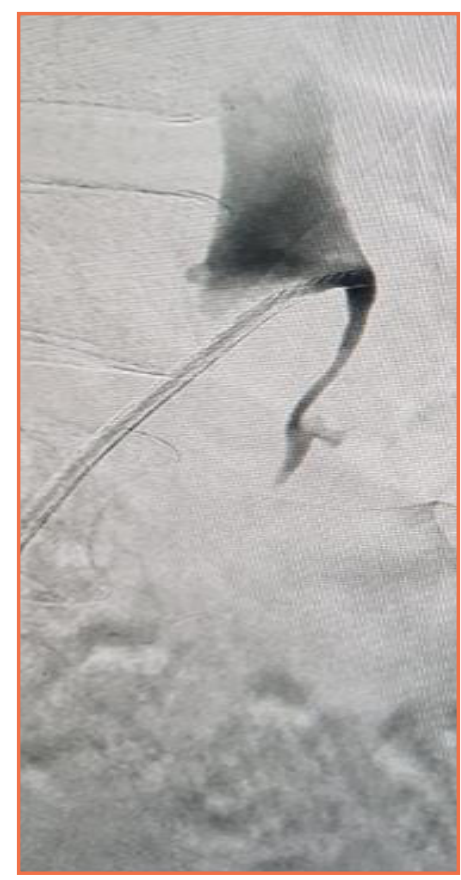

Figure 3. Aortography showing expressive reduction of the flow after administration of contrast in mesenteric inferior artery.

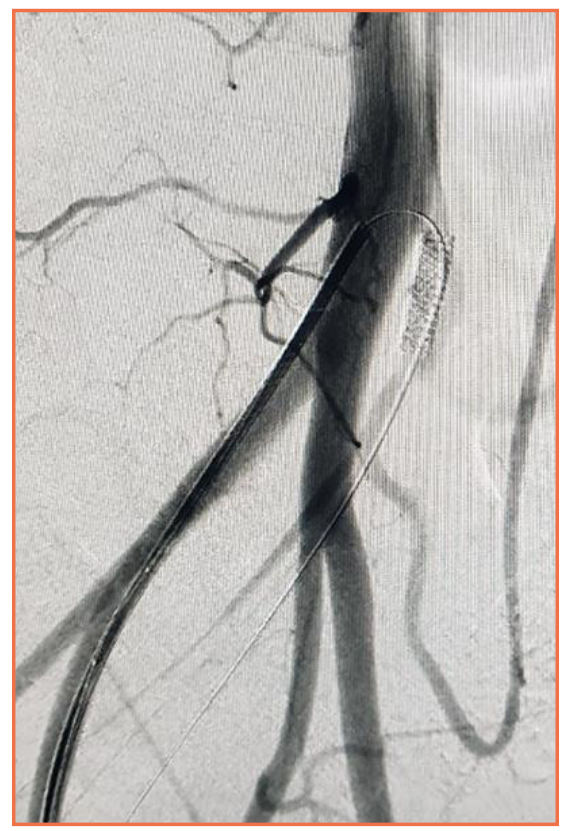

Figure 4. Aortography realized after angioplasty in mesenteric inferior artery.

\section{CONCLUSION}

Systemic vasculitis is an uncommon complication of sarcoidosis and can affect vessels of small to large caliber. This condition can mimic hypersensitivity vasculitis, polyarteritis nodosa, microscopic polyangiitis or Takayasu's arteritis, the latter being exemplified in our report. 